\title{
BRANCHING PROCESSES WITH LOCAL DEPENDENCIES
}

\author{
By Peter OlOfsson \\ Chalmers University of Technology
}

\begin{abstract}
A general multitype branching process with sibling dependencies is considered. The dependencies within a group of siblings are described by a joint probability measure, determined by the structure of that particular group. The process is analyzed by means of the embedded macro process, consisting of sibling groups. It is shown that the regular asymptotic behavior of the sibling-dependent process is guaranteed by conditions on the individual reproductions, and that these conditions are exactly the same as those normally required for an ordinary independent process that has the same individual marginals. Convergence results for the expected population size as well as the actual population size are given, and the stable population is described. The sibling-dependent process and the ordinary independent process with the same marginals are compared; some simple examples illustrate the differences and similarities. The results are extended to more general dependencies that are local in the family tree.
\end{abstract}

1. Introduction. Most branching process theory relies on some fundamental independence assumption; individuals' reproductions are independent or, in the multitype case, conditionally independent given their types. Both for theoretical reasons and from the point of view of applications it would, however, be interesting to investigate different kinds of dependencies, for instance, dependencies that are local in the family tree. In biology the term kin selection is well known, meaning that individuals put effort into helping their close relatives. This would suggest some kind of negative correlation structure between, for instance, siblings.

In cell kinetics, positive correlations between the life lengths of sister cells are well known, at least since Powell (1955). Kubitschek (1967) found similar correlations also between first cousins and second cousins. A branching process approach to sister correlations was performed by Crump and Mode (1969); the model in that paper did not, however, permit further dependencies. In Broberg (1987), general single-type branching processes with a special kind of sibling dependency are treated. Olofsson (1994), on which this paper is based, deals with general local dependencies in multitype populations. For an overview of some principal problems with dependencies in general, the reader is referred to Jagers (1995).

Received July 1994; revised October 1995.

AMS 1991 subject classifications. Primary 60J80; secondary 60F25.

Key words and phrases. General branching process, multitype, sibling dependencies, local dependencies, macro process, stable population. 
In this paper we first deal with a general multitype process with sibling dependencies and then extend the results to more general dependencies that are local in the family tree.

To make it reasonably self-contained, before dealing with the dependencies, we give a quick description of general multitype branching processes; for a comprehensive treatment the reader is referred to Jagers $(1989,1992)$.

A possible life career, $\omega$, of an individual is an element of the life space $(\Omega, \mathscr{A})$. Individual properties of interest are then considered as random elements on the life space. Fundamental such elements are the consecutive ages at childbearing $\tau(1) \leq \tau(2) \leq \cdots \leq \infty$, where $\tau(i)(\omega)$ is the age of an individual with life career $\omega$ when she begets her $i$ th child, $\tau(i)$ being $\infty$ if the individual begets less than $i$ children.

At birth each individual gets a type from the type space $(S, \mathscr{S})$, where the $\sigma$-algebra $\mathscr{S}$ is assumed countably generated. The $i$ th child of an individual with life career $\omega$ gets type $\sigma(i)(\omega)$. The birth times and types together form the reproduction process

$$
\xi(\omega, A \times B)=\#\{i: \sigma(i)(\omega) \in A, \tau(i)(\omega) \in B\},
$$

where $A \in \mathscr{S}$ and $B \in \mathscr{B}$, the Borel $\sigma$-algebra. The notation $\xi(\omega)$ and $\xi(A \times B)$ will be used depending on the context.

Identify individuals by descent: 0 denotes the ancestor, the individual $\left(x_{1}, \ldots, x_{n}\right)$ is the $x_{n}$ th child of the $x_{n-1}$ th child of $\ldots$ of the $x_{1}$ th child of the ancestor. Hence an individual is an element of the Ulam-Harris space

$$
I=\bigcup_{k=0}^{\infty} N^{k} .
$$

The notation $x y$ is used for the individual whose first coordinates are those of $x$, the last those of $y$. For instance, $x k, k=1,2, \ldots$, are $x$ 's children. The population space is then $S \times \Omega^{I}$, an outcome of which consists of the ancestor's type and a life career for each individual in $I$. The projection $U_{x}$ : $S \times \Omega^{I} \rightarrow \Omega$ singles out the life career of the individual $x$ and enables us to lift entities defined on the life space into the population space. For instance, the reproduction process of an individual $x$ is defined through

$$
\xi_{x}=\xi \circ U_{x}
$$

other random elements being defined similarly. An element of the population space is denoted $\left(s, \omega_{I}\right)$, and generally the notation $\omega_{M}$ is used for the set of lives of the individuals in $M \subseteq I$. In particular, $\omega_{x}=\omega_{I} \circ U_{x}$, the life of the individual $x$.

Assume a set of probability kernels $\{P(r, \cdot): r \in S\}$ on $\Omega$ is given and that an individual of type $s$ chooses life career according to the kernel $P(s, \cdot)$ independently of all other individuals. For each starting type $s \in S$, there is then a unique probability measure $P_{s}$ on $S \times \Omega^{I}$.

To count or measure the population, random characteristics are introduced. These are random processes

$$
\chi: S \times \Omega^{I} \times R \rightarrow R_{+},
$$


where $\chi\left(s, \omega_{I}, t\right)$ gives the contribution at age $t$ of an $s$-type individual with life and progeny's lives described by $\omega_{I}$. We assume that $\chi$ vanishes for negative times $t$. A simple example of such a characteristic is $\chi(t)=1_{R_{+}}(t)$ which is 1 if $t \geq 0$, that is, if you are born, and 0 otherwise. Note that $\chi$ may depend on an individual's whole progeny.

With $\tau_{x}$ denoting the birth time of the individual $x$ and $\chi_{x}\left(t-\tau_{x}\right)$ denoting the $\chi$-value of the individual $x$ at age $t-\tau_{x}$, the $\chi$-counted population is defined as

$$
z_{t}^{\chi}=\sum_{x \in I} \chi_{x}\left(t-\tau_{x}\right)
$$

adding the contributions of all individuals born up to time $t$ at their proper ages. The characteristic $1_{R_{+}}$mentioned above will then count the number of individuals born up to time $t$. The main objective of general branching processes is to study the asymptotics of $E_{s}\left[z_{t}^{\chi}\right]$, the expectation of the $\chi$-counted population with respect to $P_{s}$, as well as of $z_{t}^{\chi}$ itself.

2. Why sibling dependencies? In this section we give a few simple examples which illustrate some of the principal problems that arise from sibling dependencies. The basic idea of how to deal with the dependencies is also described and developed in a Galton-Watson context. In later sections this will all be done at a much more general, but also less accessible level, so this section serves the purpose of giving the reader some insight about what is going on. A first example will show what impact sibling dependencies have on three standard issues in branching process theory: extinction, growth and composition.

ExAMPLE 2.1. Consider a sibling-dependent Galton-Watson process where an individual can beget zero or three children, and where the dependencies are such that, in a group of three siblings, two will always reproduce while the third never will. All individuals are equally likely to be among the reproducing ones. Thus there is a joint probability measure on $\{0,3\}^{3}$ which gives equal probabilities to the points $(0,3,3),(3,0,3)$ and $(3,3,0)$ and has the marginals $p_{0}=1 / 3$ and $p_{3}=2 / 3$. Clearly, there are sibling dependencies; if we, for instance, know that an individual has no children, we also know that her sisters have three children each.

Let us now compare this process to an ordinary independent GaltonWatson process which has the same individual marginals. The expected number of children is 2 , and there is a positive probability of nonextinction, in which case the generation size tends to $\infty$. Indeed, the extinction probability may be computed explicitly as the smallest nonnegative solution of the equation $s=1 / 3+(2 / 3) s^{3}$, which turns out to be $(\sqrt{3}-1) / 2$.

The dependent process will have a deterministic generation size of $3 \cdot 2^{n}$ individuals in the $n$th generation (if it starts from a full group of siblings) and hence it never becomes extinct. In this example, introducing sibling dependencies thus brings the extinction probability from $(\sqrt{3}-1) / 2$ down to 0 ! 
To describe the growth rates, let $z_{n}$ be the number of individuals in the $n$th generation in the independent process and $\zeta_{n}$ the corresponding variable in the dependent process. If the processes both start from a group of three siblings, we have, for some random variable $w$,

$$
\frac{z_{n}}{2^{n}} \rightarrow w
$$

which follows from classical results for Galton-Watson processes, and

$$
\frac{\zeta_{n}}{2^{n}} \equiv 3
$$

so that the growth rates are the same in the two processes. Another wellknown fact from classical theory is that although the population size tends to $\infty$ (if extinction is avoided), the composition stabilizes in the sense that proportions of individuals with various properties converge to constants as $n \rightarrow \infty$. This fact is made precise in a quite general context in the so-called stable population, described in Jagers (1992). Having explored extinction and growth, let us finally see how the asymptotic composition may be affected by sibling dependencies.

Let $A$ be the event that an individual has no children and define $z_{n}^{A}$ and $\zeta_{n}^{A}$ as the numbers of individuals without children in the independent population and the dependent population, respectively. The probability of $A$ is $1 / 3$ in both populations and hence

$$
\frac{z_{n}^{A}}{z_{n}} \rightarrow \frac{1}{3}
$$

and

$$
\frac{\zeta_{n}^{A}}{\zeta_{n}} \equiv \frac{1}{3} .
$$

The asymptotic proportion of childless individuals is thus the same in the two populations. Now let $B$ be the event that an individual has no grandchildren. In the dependent population $B$ is obviously the same event as $A$, whereas in the independent population $B$ has probability $1 / 3+(2 / 3)(1 / 3)^{3}=29 / 81$. Hence

$$
\frac{z_{n}^{B}}{z_{n}} \rightarrow \frac{29}{81},
$$

so that the asymptotic proportion of individuals without grandchildren is not the same in the two populations. A more drastic example is to consider individuals who have children but no grandchildren since such individuals do not exist at all in the dependent population, but appear with probability $2 / 81$ in the independent population.

It is also interesting to note that although the distribution of the number of grandchildren obviously differs, the expected number of grandchildren remains the same in the two populations. 
We have seen an example where sibling dependencies affect the extinction probability, as compared with an independent process, but the growth rate remains the same. The asymptotic proportion of individuals without children is the same, whereas the asymptotic proportion differs if instead individuals without grandchildren are considered. This example illustrates the general objectives of this paper: to show that the growth rate is never affected by sibling, or more general local, dependencies, that the asymptotic composition remains the same when individual properties are considered (actually more than this holds - see Section 12), but that this composition may differ for other properties. We will also show that no extra conditions are required because of the dependencies; the conditions are all on the individual marginal reproductions and are thus the same as in an ordinary independent process that has these marginals.

The basic idea for a theory that allows sibling dependencies is to embed another process, the macro process, into the sibling-dependent process. This macro process, introduced in Broberg (1987), consists of sibling groups, to be called macro individuals. The point is, of course, that while individuals do not reproduce independently, macro individuals do, since the only dependencies are within the sibling groups.

Generally, if it is assumed that the reproduction and dependence structure of a sibling group of size $k$ is described by a joint probability measure $P(k, \cdot)$ on $N^{k}$, the macro process may be considered as multitype, the type of a macro individual being the number of siblings in that group.

Hence classical theory for multitype Galton-Watson processes applies to the macro process. Since what is interesting is still the individual process, this whole setting would be useless if it were not possible to count individuals by instead counting macro individuals. But, indeed, with $\zeta_{n}$ denoting the number of individuals in the $n$th generation and $Z_{n}(k)$ denoting the number of macro individuals of type $k$ of the $n$th generation, it holds that

$$
\zeta_{n}=\sum_{k=1}^{\infty} k Z_{n}(k)
$$

which in Example 2.1, of course, reduces to $\zeta_{n}=3 Z_{n}(3)$.
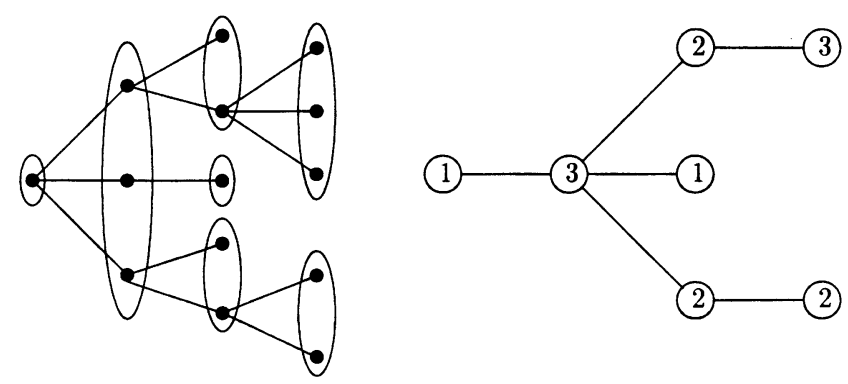

FIG. 1. A Galton-Watson process with encircled sibling groups and the corresponding macro process with its types. 
EXAMPLE 2.2. Consider a population where an individual can beget 0,1 or 2 children. In a group of 2 , one individual always splits into 2 , the other begets either 0 or 1 child with equal probabilities. The 2 siblings are equally likely to be the splitting one. A single individual begets 0,1 or 2 children with probabilities $17 / 32,8 / 32$ and $7 / 32$, respectively. In the setting above we thus have the joint probability measures $P(k, \cdot), k=1,2$, given by

$$
P(1,0)=17 / 32, \quad P(1,1)=8 / 32, \quad P(1,2)=7 / 32
$$

and

$$
P(2,(0,2))=P(2,(1,2))=P(2,(2,0))=P(2,(2,1))=1 / 4 .
$$

The macro process has type space $\{1,2\}$ and reproduction matrix

$$
M=\left[\begin{array}{cc}
8 / 32 & 7 / 32 \\
1 / 2 & 1
\end{array}\right]
$$

where the entry $(i, j)$ is the expected number of $j$-type macro children of an $i$-type macro mother. The growth rate is determined by the spectral radius of $M$, that is, $M$ 's largest eigenvalue, denoted by $\rho$. Solving the characteristic equation for $M$ yields $\rho=9 / 8$, so that we have

$$
Z_{n}(k) \sim(9 / 8)^{n} W(k),
$$

where the $W(k)$ are random variables for $k=1,2$. Again, we wish to compare with the corresponding independent process that has the same marginals. Now an individual's reproduction law depends on whether she has a sibling or not, so actually the individual process is also multitype with type space $\{1,2\}$. The type of an individual is the number of siblings in her sibling group. The reproduction matrix for the ordinary independent multitype GaltonWatson process we want to compare with is

$$
m=\left[\begin{array}{cc}
8 / 32 & 14 / 32 \\
1 / 4 & 1
\end{array}\right]
$$

which also has $\rho=9 / 8$; that is, the two processes have the same growth rates. (The extinction probabilities are again different; the dependent process will, for instance, never die out if it starts from a 2-group.)

Indeed, if we have a population where an individual can beget at most $r$ children and the sibling dependencies are described by the measures $P(k, \cdot)$, $k=1, \ldots, r$, with marginals

$$
p_{i}(j)=\text { the probability that an individual of type } i \text { begets } j \text { children, }
$$

we will have $M_{i j}=i p_{i}(j)$ and $m_{i j}=j p_{i}(j)$ for the macro process and the individual process, respectively (recall that the type of an individual is the number of individuals in her sibling group; i.e., if an individual begets $j$ children, they will all be of type $j$ ). The relation

$$
M_{i j}=\frac{i}{j} m_{i j}
$$


ensures that $\operatorname{det}(M-\lambda I)=\operatorname{det}(m-\lambda I)$, so that $M$ and $m$ actually have the same eigenvalues and hence the same spectral radii. Another condition for the convergence of multitype Galton-Watson processes is that the reproduction matrix is positive, that is, that some power of it has all entries positive. From (2.1) it follows that

$$
M_{i j}^{n}=\frac{i}{j} m_{i j}^{n}
$$

for all $n$ and positivity is equivalent for the two matrices. Again, we see that the only conditions needed are on the individual marginals and that no further conditions must be inferred due to the dependencies.

In the last example we assumed that the individual marginals are the same within each sibling group. This may be a natural assumption for splitting populations but is by no means necessary and for more general reproduction patterns is clearly unsatisfactory. If the reproduction takes place according to some point process, it would obviously be desirable to allow different individuals to have different marginals also within the same sibling group. It would also be desirable to let the whole reproduction process, rather than just the number of siblings, determine the reproduction and dependence structure. The construction above suggests that, in such a case, the type of a macro individual should be the particular realization of the reproduction process that describes that sibling group, and the type of an individual the particular realization of the reproduction process that she was born in, together with her rank. In the next section we will start building a theory for general multitype branching processes with sibling dependencies.

3. The macro process. Now consider a general multitype population where individuals who are siblings have dependent reproductions, whereas individuals who are not siblings reproduce independently of each other.

To describe the sibling dependencies, assume that the individuals born in a particular realization, $\gamma$, of a point process on $S \times R_{+}$choose their lives according to a joint probability measure $P(\gamma, \cdot)$ on $\left(\Omega^{\gamma\left(S \times R_{+}\right)}, \mathscr{A}^{\gamma\left(S \times R_{+}\right)}\right)$, independently of all other individuals. For the sake of convenience, view $P(\gamma, \cdot)$ as a measure on $\left(\Omega^{\infty}, \mathscr{A}^{\infty}\right)$, simply by choosing the $\omega_{k}$ in some arbitrary way for $k>\gamma\left(S \times R_{+}\right)$. The process of interest will be that of individuals starting from one group of siblings, with dependencies described by the $P(\gamma, \cdot)$. As in the previous section, call sibling groups macro individuals.

Let the life of a macro individual simply be the vector of the individual lives of that particular group of siblings. A macro individual's life is thus an element in $\bar{\Omega}=\Omega^{\infty}$ and of the form $\bar{\omega}=\left(\omega_{k}\right)_{k=1}^{\infty}$. Let the type of macro individual be the realization that describes how that particular group of siblings was born. Therefore, with $\mathscr{N}\left(S \times R_{+}\right)$denoting the set of realizations of point processes (or counting measures) on $S \times R_{+}$, take the type space $\Gamma$ to be

$$
\left\{\gamma \in \mathscr{N}\left(S \times R_{+}\right): 0<\gamma\left(S \times R_{+}\right)<\infty\right\},
$$


equipped with some suitable $\sigma$-algebra $\mathscr{G}$. Thus we rule out the possibility of infinitely many children, which is a natural restriction; see, for instance, Theorem 3 in Jagers (1989). Of course, the condition $\gamma\left(S \times R_{+}\right)>0$ just means that we disregard empty groups of siblings. Following the construction in Section 1 , the macro population space is $\left(\Gamma \times \bar{\Omega}^{I}, \mathscr{G} \times \overline{\mathscr{A}}^{I}\right)$, an outcome of which is denoted $\left(\gamma, \bar{\omega}_{I}\right)$ and consists of the type of the macro ancestor and the lives of all conceivable macro individuals. The set $\{P(\gamma, \cdot), \gamma \in \Gamma\}$ now defines a unique probability measure $P_{\gamma}(\cdot)$ on $\left(\Gamma \times \bar{\Omega}^{I}, \mathscr{G} \times \overline{\mathscr{A}}^{I}\right)$ for each starting type $\gamma \in \Gamma$.

Individuals thus choose lives according to the marginals of $P_{\gamma}$. Generally, the $i$ th individual in a group of siblings with type $\gamma$ chooses her life according to the marginal

$$
\tilde{Q}_{i}(\gamma, A)=\int_{\bar{\Omega}} 1_{A}\left(\omega_{i}\right) \tilde{P}(\gamma, d \bar{\omega})
$$

for $A \in \mathscr{A}$. We will assume that an individual's marginal reproduction only depends on her type, so if the $i$ th point in $\gamma$ is $\left(\sigma_{i}(\gamma), \tau_{i}(\gamma)\right)$ we have

$$
\tilde{Q}_{i}(\gamma, \cdot)=\tilde{Q}\left(\sigma_{i}(\gamma), \cdot\right)
$$

where $\{\tilde{Q}(s, \cdot), s \in S\}$ is a set of probability kernels. This means that, given an individual's type, her marginal reproduction does not depend on anything that has to do with her siblings. There are still dependencies between the actual reproductions though, so that, given some information about your siblings' number of children, for instance, your marginals will change. This assumption is actually not a restriction since we can always redefine the types to consist of the pairs $(\gamma, i)$.

As will be seen later, it is convenient to transform $\tilde{Q}$ into a probability measure on $\Gamma$, so we define

$$
Q(s, A)=\tilde{Q}(s,\{\xi \in A\} \mid\{\xi \in \Gamma\}), \quad A \in \mathscr{G} .
$$

The population of individuals started from a single individual ancestor of type $s$ then follows the marginal

$$
\tilde{Q}_{s}\left(d \omega_{I}\right)=P_{\xi\left(\omega_{0}\right)}\left(d \bar{\omega}_{I}\right) Q\left(s, d \omega_{0}\right) .
$$

[Recall that $\omega_{0}$ is the individual ancestor's life and $\omega_{I}=\left(\omega_{0}, \bar{\omega}_{I}\right)$ since the macro process starts from the children of the individual ancestor.]

A macro individual is considered born with the individual mother of that group of siblings if she begets any children. Then we can define birth times for the macro individual recursively:

$$
\begin{aligned}
\tau_{0}^{\prime} & = \begin{cases}0, & \text { if } \gamma\left(S \times R_{+}\right)>0, \\
\infty, & \text { otherwise, }\end{cases} \\
\tau_{x i}^{\prime} & = \begin{cases}\tau_{x}^{\prime}+\tau_{i}\left(\xi_{x}\right), & \text { if } \xi_{x i}\left(S \times R_{+}\right)>0, \\
\infty, & \text { otherwise. }\end{cases}
\end{aligned}
$$

Note that by this we do not allow empty groups of siblings to be born in finite time. We also see that if the individual $x$ begets any children, then $\tau_{x}^{\prime}=\tau_{x}$. 
The macro reproduction process becomes

$$
\eta(\gamma, \bar{\omega}, A \times[0, t])=\#\left\{i: \xi\left(\omega_{i}\right) \in A, \tau_{i}(\gamma) \leq t\right\}
$$

where $A \in \mathscr{S}$ and $t \geq 0$.

The macro reproduction kernel, that is, the expectation of $\eta$ with respect to the measure $\tilde{P}(\gamma, \cdot)$, is then

$$
\begin{aligned}
M(\gamma, A \times[0, t]) & =E_{\gamma}[\eta(A \times[0, t])] \\
& =\sum_{i=1}^{\infty} Q\left(\sigma_{i}(\gamma), A\right) 1_{\left\{\tau_{i}(\gamma) \leq t\right\}},
\end{aligned}
$$

the number of macro individuals with types in $A$ born before $t$. Hence, with $\delta_{x}$ denoting the unit point mass in $x$,

$$
M\left(\gamma, d \gamma^{\prime} \times d t\right)=\sum_{i=1}^{\infty} Q\left(\sigma_{i}(\gamma), d \gamma^{\prime}\right) \delta_{\tau_{i}(\gamma)}(d t) .
$$

EXAMPLE 3.1. As a special case we mention the model of Crump and Mode (1969). They consider a Bellman-Harris process (i.e., a process where individuals have random lifespans and reproduce by splitting at death) that allows for sibling dependencies in the following way. The lifespans $L_{1}, \ldots, L_{k}$ of a collection of $k$ siblings are assumed to be exchangeable and have joint distribution function $G_{k}$. The probability that these siblings have $n_{1}, \ldots, n_{k}$ offspring is $p_{n_{1}, \ldots, n_{k}}$; also the numbers of children are assumed exchangeable. Otherwise the independence assumptions of the Bellman-Harris process hold. Denote the one-dimensional marginal of $G_{k}$ by $F_{k}$ and the probability that an individual in a $k$-group begets $j$ children by $p_{k}(j)$.

A macro type thus consists of a lifespan and a number of children. If $\gamma=(l, k)$ and $A=[0, u] \times\{j\}, u \geq 0, j \geq 1,(3.2)$ yields

$$
M(\gamma, A \times[0, t])=k F_{k}(u) p_{k}(j) 1_{\{l \leq t\}},
$$

since $\tau_{i}(\gamma)=l$ for $i=1,2, \ldots, k$.

So now a macro process has been constructed; it is an ordinary multitype branching process but would not be of much use unless it could measure the same aspects of the population as the individual, sibling-dependent, process does. The next objective is to show that the macro process is indeed useful in this way. Recall the $\chi$-counted population from Section 1 . We will show that there exists a macro characteristic $\chi^{\prime}$ that measures exactly the same thing as the individual characteristic $\chi$. This macro characteristic is defined through

$$
\chi^{\prime}(t)=\sum_{i=1}^{\infty} \chi_{i}\left(t-\tau_{i}\right)
$$

where

$$
\chi_{i}\left(\gamma, \bar{\omega}_{I}, t-\tau_{i}\right)=\chi\left(\sigma_{i}(\gamma), \omega_{i I}, t-\tau(\gamma)\right),
$$

$\omega_{i I}$ denoting the lives of the individuals in the set $i I=\{i x\}_{x \in I}$. Hence the macro characteristic adds the contributions of the individuals in each sibling group and the following lemma is obvious. 
Lemma 3.1. Let $\chi^{\prime}$ be defined through (3.3) and

Then

$$
Z_{t}^{\chi^{\prime}}=\sum_{x \in I} \chi_{x}^{\prime}\left(t-\tau_{x}^{\prime}\right)
$$

$$
z_{t}^{\chi}=\chi_{0}(t)+Z_{t}^{\chi^{\prime}}
$$

Note that $Z_{t}^{\chi^{\prime}}$ starts counting from the children of the ancestor.

4. Some Markov renewal theory. The asymptotics of the expected population size are analyzed by means of Markov renewal theory, and we will give the definitions needed for this purpose, following Shurenkov (1992), which is an English version of Shurenkov (1989). Let $(E, \mathscr{E})$ be a measurable space with $\mathscr{E}$ countably generated and $K(x, d y \times d t)$ a nonnegative kernel on $\left(E \times R_{+}, \mathscr{E} \times \mathscr{B}\right), x \in E$ and $\mathscr{B}$ the Borel $\sigma$-algebra on $R_{+}$.

For any real $\lambda$ define $\hat{K}_{\lambda}(x, d y)$ through

and

$$
\hat{K}_{\lambda}(x, d y)=\int_{0}^{\infty} e^{-\lambda t} K(x, d y \times d t)
$$

$$
\hat{K}_{\lambda}^{n}(x, d y)=\int_{E} \hat{K}_{\lambda}^{n-1}(x, d z) \hat{K}_{\lambda}(z, d y) .
$$

By convention we put

$$
\hat{K}_{\lambda}^{0}(x, d y)=\delta_{x}(d y),
$$

placing a unit point mass at $x$.

A kernel $\hat{K}_{\lambda}$ is called $\sigma$-finite if there exists an $\mathscr{E} \times \mathscr{E}$-measurable, strictly positive function $f$ such that

$$
\int_{E} f(x, y) \hat{K}_{\lambda}(x, d y)<\infty
$$

for each $x \in E$.

Definition 4.1. The Perron root of $\hat{K}_{\lambda}$, denoted by $\rho\left(\hat{K}_{\lambda}\right)$, is defined through

$$
\frac{1}{\rho\left(\hat{K}_{\lambda}\right)}=\sup \left\{c \geq 0: \sum_{n \geq 0} c^{n} \hat{K}_{\lambda}^{n} \text { is } \sigma \text {-finite }\right\} .
$$

Definition 4.2. The kernel $\hat{K}_{\lambda}$ is called conservative if there exists a $\sigma$-finite nonzero measure $m$ on $(E, \mathscr{E})$ such that

$$
m(A)>0 \Rightarrow \sum_{n \geq 0} \frac{1}{\rho^{n}} \hat{K}_{\lambda}^{n}(x, A)=\infty, \quad A \in \mathscr{E},
$$

for all $x \in E$.

5. Mean convergence. Since the expectation of $z_{t}^{\chi}$ is the expectation of a sum, it will not be affected by the sibling dependencies. The asymptotic results that can be obtained are valid since $E_{s}\left[z_{t}^{\chi}\right]$, the expectation of $z_{t}^{\chi}$ 
when the ancestor has type $s$, satisfies a Markov renewal equation, sibling dependencies or not. These asymptotics are determined by the reproduction kernel $\mu$, defined as

$$
\mu(s, A \times[0, t])=E_{s}[\xi(A \times[0, t])],
$$

the expected number of children with types in $A$ an $s$-type individual begets before age $t$. The Malthusian parameter, $\alpha$, is now defined so that $\hat{\mu}_{\alpha}$ has Perron root 1 . Once $\alpha$ has been fixed we omit it in the subscript, writing $\hat{\mu}$ rather than $\hat{\mu}_{\alpha}$. We only consider the supercritical case, that is, when $\alpha>0$.

If the kernel $\hat{\mu}$ is conservative it determines an invariant function $h$ and an invariant measure $\pi$ such that

$$
h(s)=\int_{S} h(r) \hat{\mu}(s, d r)
$$

and

$$
\pi(d s)=\int_{S} \hat{\mu}(r, d s) \pi(d r)
$$

with the interpretations that $h(s)$ is the reproductive value of the type $s$ and $\pi$ the stable-type distribution. To guarantee that $\pi$ can be normed to a probability measure, the assumptions $h \in L^{1}[\pi]$ and inf $h>0$ are made. If there exists an $a>0$ such that

$$
\sup _{s} \mu(s, S \times[0, a])<1
$$

and the stable age of child-bearing, $\beta$, defined through

$$
\beta=\int_{S \times S \times R_{+}} e^{-\alpha t} h(r) \mu(s, d r \times d t) \pi(d s)
$$

is positive and finite, the process is called strictly Malthusian.

The kernel $\mu$ is also assumed nonlattice, latticeness meaning that there exists a number $d>0$ and a function $c: E \rightarrow[0, d]$ such that $\mu$ is concentrated on the lattice

$$
\left\{(t, r) \in R_{+} \times S: t=c(s)-c(r)+n d \text { for some } n=0,1, \ldots\right\}
$$

for $\pi$-almost all $s \in S$.

Finally, an integrability requirement on $\chi$ is needed and for that purpose we give the following definition:

Definition 5.1. A function $g: S \times R_{+} \rightarrow R_{+}$is called directly Riemann integrable $(\pi)$ if

and

$$
\sum_{n \geq 0} \int_{S} \sup _{n \leq t \leq n+1} g(s, t) \pi(d s)<\infty
$$

$\delta \sum_{n \geq 0} \int_{S}\left(\sup _{n \delta \leq t \leq(n+1) \delta} g(s, t)-\inf _{n \delta \leq t \leq(n+1) \delta} g(s, t)\right) \pi(d s) \rightarrow 0 \quad$ as $\delta \rightarrow 0+$. 
Under these conditions the general Markov renewal theorem of Shurenkov (1992) can be applied. Let $\hat{\chi}(\alpha)=\int_{0}^{\infty} \alpha e^{-\alpha t} \chi(t) d t$ and let $E_{\pi}$ denote expectation when the type is not fixed but distributed according to $\pi$.

THEOREM 5.2. Consider a nonlattice, strictly Malthusian branching process with sibling dependencies. Let $\chi$ be bounded and the function $e^{-\alpha t} E_{s}[\chi(t)]$ directly Riemann integrable. Then

$$
E_{s}\left[e^{-\alpha t} z_{t}^{\chi}\right] \rightarrow \frac{E_{\pi}[\hat{\chi}(\alpha)]}{\alpha \beta} h(s)
$$

as $t \rightarrow \infty$ for $\pi$-almost all $s \in S$.

Proof. As for Theorem 3 in Jagers (1992).

Note that the theorem looks exactly as its independent analog. One way of expressing this is that introducing sibling dependencies qualitatively does not change the asymptotics. The difference is rather quantitative, since the constant $E_{\pi}[\hat{\chi}(\alpha)]$ may differ (recall Example 2.1). When we turn to the asymptotics of $z_{t}^{\chi}$ itself ( $L^{1}$-convergence is what will be considered), things will be more complicated. We will then work directly with the macro process and must for that purpose explore how the individual process and the macro process relate to each other.

6. The Malthusian parameter. To investigate the Markov renewal properties, a connection between $\hat{\mu}_{\lambda}^{n}$ and $\hat{M}_{\lambda}^{n}$ needs to be established. First note that

$$
\begin{aligned}
\hat{\mu}_{\lambda}(s, d r) & =\int_{0}^{\infty} e^{-\lambda t} E_{s}[\xi(d r \times d t)] \\
& =\sum_{i=1}^{\infty} \int_{\Gamma} \exp \left(-\lambda \tau_{i}\left(\gamma^{\prime}\right)\right) \delta_{\sigma_{i}\left(\gamma^{\prime}\right)}(d r) Q\left(s, d \gamma^{\prime}\right)
\end{aligned}
$$

and, by (3.2),

$$
\hat{M}\left(\gamma, d \gamma^{\prime}\right)=\sum_{i=1}^{\infty} \exp \left(-\alpha \tau_{i}(\gamma)\right) Q\left(\sigma_{i}(\gamma), d \gamma^{\prime}\right),
$$

which yields the connection

$$
\hat{M}_{\lambda}^{n}\left(\gamma, d \gamma^{\prime}\right)=\sum_{i=1}^{\infty} \int_{S} \exp \left(-\lambda \tau_{i}(\gamma)\right) \hat{\mu}_{\lambda}^{n-1}\left(\sigma_{i}(\gamma), d s\right) Q\left(s, d \gamma^{\prime}\right)
$$

for $n \geq 1$, between $\hat{M}_{\lambda}$ and $\hat{\mu}_{\lambda}\left[\hat{M}_{\lambda}^{0}\left(\gamma, d \gamma^{\prime}\right)=\delta_{\gamma}\left(d \gamma^{\prime}\right)\right]$. Now it is possible to prove that $\hat{\mu}_{\lambda}$ and $\hat{M}_{\lambda}$ define the same Malthusian parameter. 
Lemma 6.1. The reproduction kernel $\hat{\mu}_{\lambda}$ has Perron root 1 if and only if $\hat{M}_{\lambda}$ has Perron root 1.

PRoOF. We will show that $\rho\left(\hat{\mu}_{\lambda}\right)=\rho\left(\hat{M}_{\lambda}\right)$ for any real $\lambda$. First let $c<$ $1 / \rho\left(\hat{\mu}_{\lambda}\right)$. Then there exists a function $f>0$ such that

$$
\sum_{n \geq 0} c^{n} \int_{S} f(s, r) \hat{\mu}_{\lambda}^{n}(s, d r)<\infty
$$

where, by (6.1),

$$
\begin{aligned}
& \int_{S} f(s, r) \hat{\mu}_{\lambda}^{n}(s, d r) \\
& \quad=\sum_{j=1}^{\infty} \int_{S \times \Gamma} \exp \left(-\lambda \tau_{j}\left(\gamma^{\prime}\right)\right) f\left(s, \sigma_{j}\left(\gamma^{\prime}\right)\right) \hat{\mu}_{\lambda}^{n-1}(s, d r) Q\left(r, d \gamma^{\prime}\right)
\end{aligned}
$$

for $n \geq 1$ and

$$
\int_{S} f(s, r) \hat{\mu}_{\lambda}^{0}(s, d r)=f(s, s) .
$$

The question is if there exists a function $F>0$ such that

$$
\sum_{n \geq 0} \int_{\Gamma} F\left(\gamma, \gamma^{\prime}\right) \hat{M}_{\lambda}^{n}\left(\gamma, d \gamma^{\prime}\right)<\infty
$$

for all $\gamma \in \Gamma$. Therefore, take a $\gamma \in \Gamma$, fix an $i \leq \gamma\left(S \times R_{+}\right)$and note that, by (6.3) and (6.4),

$$
\sum_{n \geq 0} c^{n} \sum_{j=1}^{\infty} \int_{S \times \Gamma} \exp \left(-\lambda \tau_{j}\left(\gamma^{\prime}\right)\right) f\left(\sigma_{i}(\gamma), \sigma_{j}\left(\gamma^{\prime}\right)\right) \hat{\mu}_{\lambda}^{n}\left(\sigma_{i}(\gamma), d r\right) Q\left(r, d \gamma^{\prime}\right)<\infty
$$

and hence also

$$
\begin{gathered}
\sum_{n \geq 0} c^{n} \sum_{i=1}^{\infty} \sum_{j=1}^{\infty} \int_{S \times \Gamma} \exp \left(-\lambda \tau_{i}(\gamma)\right) \exp \left(-\lambda \tau_{j}\left(\gamma^{\prime}\right)\right) f\left(\sigma_{i}(\gamma), \sigma_{j}\left(\gamma^{\prime}\right)\right) \\
\times \hat{\mu}_{\lambda}^{n}(\sigma i(\gamma), d r) Q\left(r, d \gamma^{\prime}\right)<\infty
\end{gathered}
$$

Now define

$$
F_{i}\left(\gamma, \gamma^{\prime}\right)=\sum_{j=1}^{\infty} \exp \left(-\lambda \tau_{j}\left(\gamma^{\prime}\right)\right) f\left(\sigma_{i}(\gamma), \sigma_{j}\left(\gamma^{\prime}\right)\right)
$$

so that

$$
\sum_{n \geq 0} c^{n} \sum_{i=1}^{\infty} \int_{S \times \Gamma} \exp \left(-\lambda \tau_{i}(\gamma)\right) F_{i}\left(\gamma, \gamma^{\prime}\right) \hat{\mu}_{\lambda}^{n}\left(\sigma_{i}(\gamma), d r\right) Q\left(r, d \gamma^{\prime}\right)<\infty .
$$

Since for each $\gamma \in \Gamma$ there are a finite number of $F_{i}, i=1,2, \ldots, \gamma(S \times R+)<$ $\infty$, we can define

$$
F\left(\gamma, \gamma^{\prime}\right)=\min _{i=1,2, \ldots, \gamma(\infty)} F_{i}\left(\gamma, \gamma^{\prime}\right)
$$


and, by (6.2), obtain that

$$
\begin{aligned}
\sum_{n \geq 0} c^{n} \int_{\Gamma} F\left(\gamma, \gamma^{\prime}\right) \hat{M}_{\lambda}^{n}\left(\gamma, d \gamma^{\prime}\right) \\
\quad=F(\gamma, \gamma)+\sum_{n \geq 1} c^{n} \sum_{i=1}^{\infty} \int_{S \times \Gamma} \exp \left(-\lambda \tau_{i}(\gamma)\right) F\left(\gamma, \gamma^{\prime}\right) \hat{\mu}_{\lambda}^{n-1}\left(\sigma_{i}(\gamma), d r\right) Q\left(r, d \gamma^{\prime}\right) \\
\quad \leq F(\gamma, \gamma)+\sum_{n \geq 1} c^{n} \sum_{i=1}^{\infty} \int_{S \times \Gamma} \exp \left(-\lambda \tau_{i}(\gamma)\right) F_{i}\left(\gamma, \gamma^{\prime}\right) \hat{\mu}_{\lambda}^{n-1}\left(\sigma_{i}(\gamma), d r\right) Q\left(r, d \gamma^{\prime}\right) \\
\quad<\infty .
\end{aligned}
$$

Hence $\sum_{n \geq 0} c^{n} \hat{M}_{\lambda}^{n}$ is $\sigma$-finite if $c<1 / \rho\left(\hat{\mu}_{\lambda}\right)$ and therefore $\rho\left(\hat{M}_{\lambda}\right) \geq \rho\left(\hat{\mu}_{\lambda}\right)$.

Next let $c>1 / \rho\left(\hat{\mu}_{\lambda}\right)$. For all $f>0$ there exists an $s \in S$ such that

$$
\sum_{n \geq 0} c^{n} \int_{S} f(s, r) \hat{\mu}_{\lambda}^{n}(s, d r)=\infty .
$$

The question is now if, given an $F>0$, there exists a $\gamma \in \Gamma$ such that

$$
\sum_{n \geq 0} c^{n} \int_{\Gamma} F\left(\gamma, \gamma^{\prime}\right) \hat{M}_{\lambda}^{n}\left(\gamma, d \gamma^{\prime}\right)=\infty
$$

that is,

$$
F(\gamma, \gamma)+\sum_{n \geq 0} c^{n} \sum_{i=1}^{\infty} \int_{S \times \Gamma} F\left(\gamma, \gamma^{\prime}\right) \exp \left(-\lambda \tau_{i}(\gamma)\right) \hat{\mu}_{\lambda}^{n}\left(\sigma_{i}(\gamma), d r\right) Q\left(r, d \gamma^{\prime}\right)=\infty .
$$

For an arbitrary $F>0$ let

$$
g(\gamma, r)=\int_{\Gamma} F\left(\gamma, \gamma^{\prime}\right) Q\left(r, d \gamma^{\prime}\right),
$$

which is strictly positive if we assume that all the $Q(r, \Gamma)>0$. This assumption can be made since it will later follow from the fact that $h>0$. Now let

$$
\Gamma_{1}=\left\{\gamma \in \Gamma: \gamma\left(S \times R_{+}\right)=1, \tau_{1}(\gamma)=1\right\},
$$

so that every $s \in S$ corresponds to a unique $\gamma=\gamma(s)$ in $\Gamma_{1}$. Define

$$
f(s, r)=g(\gamma(s), r) .
$$

Then there exists an $s_{0} \in S$ such that

$$
\sum_{n \geq 0} c^{n} \int_{S} f\left(s_{0}, r\right) \hat{\mu}_{\lambda}^{n}\left(s_{0}, d r\right)=\infty .
$$

Choose $\gamma_{0}=\gamma\left(s_{0}\right)$ to obtain

$$
\begin{aligned}
\sum_{n \geq 0} c^{n} \int_{\Gamma} F\left(\gamma_{0}, \gamma^{\prime}\right) \hat{M}_{\lambda}^{n}\left(\gamma_{0}, d \gamma^{\prime}\right) & \\
\quad= & F\left(\gamma_{0}, \gamma_{0}\right)+\sum_{i=1}^{\infty} \sum_{n \geq 0} c^{n} \int_{S} \exp \left(-\lambda \tau_{i}\left(\gamma_{0}\right)\right) g\left(\gamma_{0}, r\right) \hat{\mu}_{\lambda}^{n}\left(\sigma_{i}\left(\gamma_{0}\right), d r\right) \\
& =F\left(\gamma_{0}, \gamma_{0}\right)+\exp \left(-\tau_{l}\left(\gamma_{0}\right)\right) \sum_{n \geq 0} c^{n} \int_{S} f\left(s_{0}, r\right) \hat{\mu}_{\lambda}^{n}\left(s_{0}, d r\right)=\infty .
\end{aligned}
$$


It has been shown that $\sum_{n \geq 0} c^{n} \hat{M}_{\lambda}^{n}$ is not $\sigma$-finite if $c>1 / \rho\left(\hat{\mu}_{\lambda}\right)$. Therefore, $\rho\left(\hat{M}_{\lambda}\right) \leq \rho\left(\hat{\mu}_{\lambda}\right)$ and the proof is complete.

7. The invariant functions and measures. First we treat the question of conservativity.

LEMMA 7.1. The reproduction kernel $\hat{\mu}$ is conservative if and only if $\hat{M}$ is conservative.

Proof. Assume first that $\hat{\mu}$ is conservative. Then there exists a measure $m$ on $(S, \mathscr{S})$ such that

$$
m(A)>0 \Rightarrow \sum_{n \geq 0} \hat{\mu}^{n}(s, A)=\infty
$$

for each $s \in S$. We wish to find a measure $l$ on $(\Gamma, \mathscr{G})$ such that

$$
\sum_{n \geq 0} \hat{M}^{n}(\gamma, B)=\infty
$$

for all $\gamma \in \Gamma$ whenever $l(B)>0$. Such a measure is

$$
l(d \gamma) \int_{S} Q(s, d \gamma) m(d s) .
$$

To see this, consider an $A \in \mathscr{G}$ with $l(A)>0$. Then there exist a subset $B$ of $S$ and a number $c>0$ such that $m(B)>0$ and $Q(s, A) \geq c$ when $s \in B$. Therefore, for any $\gamma \in \Gamma$,

$$
\sum_{n \geq 1} \hat{M}(\gamma, A) \geq c \sum_{i=1}^{\infty} \int_{S} \exp \left(-\alpha \tau_{i}(\gamma)\right) \sum_{n \geq 0} \hat{\mu}^{n}\left(\sigma_{i}(\gamma), B\right)=\infty
$$

since $m(B)>0$.

Now assume that $\hat{M}$ is conservative. Then there exists a measure $l$ on $(\Gamma, \mathscr{G})$ such that

$$
\sum_{n \geq 0} \hat{M}^{n}(\gamma, B)=\infty
$$

for all $\gamma \in \Gamma$ whenever $l(B)>0$. A measure exhibiting $\hat{\mu}$ as conservative is

$$
m(d s)=\sum_{i=1}^{\infty} \int_{\Gamma} \exp \left(-\alpha \tau_{i}(\gamma)\right) \delta_{\sigma_{i}(\gamma)}(d s) l(d \gamma) .
$$

Indeed, if $m(B)>0$, then there exist an $A \in \mathscr{G}$ and a number $c>0$ such that $l(A)>0$ and $\sum_{i=1}^{\infty} \exp \left(-\alpha \tau_{i}(\gamma)\right) 1_{B}\left(\sigma_{i}(\gamma)\right) \geq c$ when $\gamma \in A$. Hence, by (6.1),

$$
\begin{aligned}
\sum_{n \geq 1} \hat{\mu}^{n}(s, B) & =\sum_{n \geq 0} \sum_{i=1}^{\infty} \int_{S \times \Gamma} \exp \left(-\alpha \tau_{i}(\gamma)\right) 1_{B}\left(\sigma_{i}(\gamma)\right) \hat{\mu}^{n}(s, d r) Q(r, d \gamma) \\
& \geq c \sum_{n \geq 0} \int_{S} \hat{\mu}^{n}(s, d r) Q(r, A) .
\end{aligned}
$$

But, by (6.2),

$$
\sum_{n \geq 1} \hat{M}^{n}(\gamma, A)=\sum_{n \geq 0} \sum_{i=1}^{\infty} \int_{S} \exp \left(-\alpha \tau_{i}(\gamma)\right) \hat{\mu}^{n}\left(\sigma_{i}(\gamma), d r\right) Q(r, A)=\infty
$$


for every $\gamma \in \Gamma$ since $l(A)>0$. In particular, it holds for all $\gamma$ in the set $\Gamma_{1}$ defined in (6.7). Therefore, fix an $s \in S$ and let $\gamma(s)$ be the corresponding element of $\Gamma_{1}$ so that $\gamma\left(S \times R_{+}\right)=1, \tau_{1}(\gamma(s))=1$ and $\sigma_{1}(\gamma(s))=s$. By the above,

$$
\sum_{n \geq 0} \int_{S} \hat{\mu}^{n}(s, d r) Q(r, A)=\infty
$$

and hence

$$
\sum_{n \geq 0} \hat{\mu}^{n}(s, B)=\infty
$$

for all $s \in S$ and $\hat{\mu}$ is conservative.

Hence we know that growth rate and conservativity are properties that are equivalent for the individual process and the macro process, respectively, or put another way, that there is no way to change these properties by introducing sibling dependencies into an ordinary multitype process with reproduction measure $\mu$.

We are now going to explore the connection between the two processes further. It has been shown that assuming $\hat{\mu}$ conservative implies that $\hat{M}$ also is conservative and there thus exists a function $H$ and and a measure $\psi$ such that

$$
H(\gamma)=\int_{\Gamma} H\left(\gamma^{\prime}\right) \hat{M}\left(\gamma, d \gamma^{\prime}\right)
$$

and

$$
\psi(d \gamma)=\int_{\Gamma} \hat{M}\left(\gamma^{\prime}, d \gamma\right) \psi\left(d \gamma^{\prime}\right)
$$

One might ask if more can be done than just giving an existence statement; is it possible to make use of the relation between the individual process and the macro process and give $H$ and $\psi$ explicitly in terms of $h$ and $\pi$ ? With the notation

$$
\bar{\gamma}=\int_{S \times R_{+}} e^{-\alpha t} h(s) \gamma(d s \times d t)=\sum_{i=1}^{\infty} \exp \left(-\alpha \tau_{i}(\gamma)\right) h\left(\sigma_{i}(\gamma)\right),
$$

the affirmative answer is given in the following lemma.

LEMMA 7.2. If $\hat{\mu}$ has invariant measure $\pi$ and invariant function $h$, then $\hat{M}$ has invariant measure $\psi$ and invariant function $H$ given by

$$
\begin{aligned}
H(\gamma) & =\bar{\gamma} \\
\psi(d \gamma) & =\int_{S} Q(s, d \gamma) \pi(d s) .
\end{aligned}
$$

Proof. Just insert the candidates for $H$ and $\psi$ in the defining relations (7.1) and (7.2) and make use of the invariance properties of $h$ and $\pi$. 
The expressions in Lemma 7.2 are intuitively obvious. The reproductive value of a sibling group of type $\gamma, H(\gamma)$, weighs together the reproductive values of the individuals in that group. The weights are exponentially decreasing functions of the birth times, thus taking into account that the earlier you are born, the more time you have to contribute to the population.

Further, if an individual has type $s$, then her children will be born according to the measure $Q(s, d \gamma)$, and the expression for $\psi$ follows.

It is also possible to give $h$ and $\pi$ in terms of $H$ and $\psi$, as shown in the following lemma.

LEMMA 7.3. If $\hat{M}$ has invariant measure $\psi$ and invariant function $H$, then $\hat{\mu}$ has invariant measure $\pi$ and invariant function $h$ given by

$$
\begin{aligned}
h(s) & =\int_{\Gamma} H(\gamma) Q(s, d \gamma), \\
\pi(d s) & =\sum_{i=1}^{\infty} \int_{\Gamma} \exp \left(-\alpha \tau_{i}(\gamma)\right) \delta_{\sigma_{i}(\gamma)}(d s) \psi(d \gamma) .
\end{aligned}
$$

PROOF. Insert in the defining relations and use the invariance properties of $H$ and $\psi$.

8. Convergence of the macro process. We will now show that the remaining Markov renewal conditions mentioned in Section 5 carry over from the individual process to the macro process. One such condition is latticeness. Since individuals and macro individuals are born at the same time points, it is intuitively clear that $M$ is nonlattice if $\mu$ is so. The technicalities that have to do with almost everywhere qualifications with respect to $\pi$ and $\psi$ are omitted here. They may be found in Olofsson (1994).

By (3.3), it is clear that $\chi^{\prime}$ need not be bounded even if $\chi$ is so. Neither will the condition

$$
\sup _{s} \mu(s, S \times[0, a])<1
$$

carry over to the macro process. These two conditions are, however, used only to guarantee that

$$
\sup _{t \geq 0} E_{s}\left[e^{-\alpha t} z_{t}^{\chi}\right]<\infty
$$

for all $s \in S$, a condition needed for the Markov renewal theorem. For the macro process we note that

$$
E_{\gamma}\left[e^{-\alpha t} Z_{t}^{\chi^{\prime}}\right]=\sum_{i=1}^{\infty} \exp \left(-\alpha \tau_{i}(\gamma)\right) E_{\sigma_{i}(\gamma)}\left[\exp \left(-\alpha\left(t-\tau_{i}\right)\right) z_{t-\tau_{i}}^{\chi}(i)\right]
$$

and therefore it also holds that

$$
\sup _{t \geq 0} E_{\gamma}\left[e^{-\alpha t} Z_{t}^{\chi^{\prime}}\right]<\infty
$$

for all $\gamma \in \Gamma$. 
It is quite easy to show also that the direct Riemann integrability carries over to the macro process. We state this as a lemma; the proof may be found in Olofsson (1994).

LEMMA 8.1. If $e^{-\alpha t} E_{s}[\chi(t)]$ is directly Riemann integrable $(\pi)$, then $e^{-\alpha t} E_{\gamma}\left[\chi^{\prime}(t)\right]$ is directly Riemann integrable $(\psi)$.

Since all the conditions needed for the Markov renewal theorem are satisfied, we can state the following result.

TheOREM 8.2. Assume the conditions of Theorem 5.1 and let $\chi^{\prime}$ be defined through (3.3). Then, with $B$ denoting the stable age of child-bearing in the macro process,

$$
E_{\gamma}\left[e^{-\alpha t} Z_{t}^{\chi^{\prime}}\right] \rightarrow \frac{E_{\psi}\left[\hat{\chi}^{\prime}(\alpha)\right]}{\alpha B} \bar{\gamma}
$$

as $t \rightarrow \infty$ for $\psi$-almost all $\gamma \in \Gamma$.

It can be shown that $B=\beta$ and that, if $\chi^{\prime}$ is of the form (3.3), then

$$
E_{\psi}\left[\hat{\chi}^{\prime}(\alpha)\right]=E_{\pi}[\hat{\chi}(\alpha)] \text {. }
$$

9. The $x \log x$ condition. In the analysis of the actual population, the $x \log x$ condition is of crucial importance. With

$$
\bar{\xi}=\int_{S \times R_{+}} e^{-\alpha t} h(r) \xi(d r \times d t),
$$

the $x \log x$ condition is

$$
E_{\pi}\left[\bar{\xi} \log ^{+} \bar{\xi}\right]<\infty
$$

The corresponding condition for the macro process is

$$
E_{\psi}\left[\bar{\eta} \log ^{+} \bar{\eta}\right]<\infty,
$$

where, because of the special relationship between the individual process and the macro process,

$$
\bar{\eta}=\sum_{i=1}^{\infty} \exp \left(-\alpha \tau_{i}\left(\xi_{0}\right)\right) \bar{\xi}_{i} .
$$

It will now be shown that these two conditions are actually equivalent.

LemMa 9.1. $\quad E_{\pi}\left[\bar{\xi} \log ^{+} \bar{\xi}\right]<\infty \quad \Leftrightarrow \quad E_{\psi}\left[\bar{\eta} \log ^{+} \bar{\eta}\right]<\infty$.

Proof. The proof relies on the convexity of the function $x \log ^{+} x$. First assume that $E_{\pi}\left[\bar{\xi} \log ^{+} \bar{\xi}\right]<\infty$, denote

$$
\hat{\gamma}(\alpha)=\sum_{i=1}^{\infty} \exp \left(-\alpha \tau_{i}(\gamma)\right)
$$


and note that

$$
\begin{aligned}
E_{\gamma}\left[\bar{\eta} \log ^{+} \bar{\eta}\right]= & E_{\gamma}\left[\bar{\eta} \log ^{+}\left(\sum_{i=1}^{\infty} \exp \left(-\alpha \tau_{i}(\gamma)\right) \bar{\xi}_{i}\right)\right] \\
= & E_{\gamma}\left[\bar{\eta} \log ^{+}\left(\hat{\gamma}(\alpha) \sum_{i=1}^{\infty} \frac{\exp \left(-\alpha \tau_{i}(\gamma)\right)}{\hat{\gamma}(\alpha)} \bar{\xi}_{i}\right)\right] \\
\leq & E_{\gamma}\left[\bar{\eta} \log ^{+} \hat{\gamma}(\alpha)\right] \\
& +\hat{\gamma}(\alpha) E_{\gamma}\left[\sum_{i=1}^{\infty} \frac{\exp \left(-\alpha \tau_{i}(\gamma)\right)}{\hat{\gamma}(\alpha)} \bar{\xi}_{i} \log ^{+}\left(\sum_{i=1}^{\infty} \frac{\exp \left(-\alpha \tau_{i}(\gamma)\right)}{\hat{\gamma}(\alpha)} \bar{\xi}_{i}\right)\right] \\
= & I_{1}(\gamma)+I_{2}(\gamma),
\end{aligned}
$$

since $\log ^{+} a b \leq \log ^{+} a+\log ^{+} b$. Further

$$
I_{1}(\gamma)=E_{\gamma}[\bar{\eta}] \log ^{+} \hat{\gamma}(\alpha)=\bar{\gamma} \log ^{+} \hat{\gamma}(\alpha) .
$$

Since, with $\kappa=\inf h>0$,

$$
\bar{\gamma}=\sum_{i=1}^{\infty} \exp \left(-\alpha \tau_{i}(\gamma)\right) h\left(\sigma_{i}(\gamma)\right) \geq \kappa \sum_{i=1}^{\infty} \exp \left(-\alpha \tau_{i}(\gamma)\right)=\kappa \hat{\gamma}(\alpha),
$$

we obtain

$$
\begin{aligned}
\int_{\Gamma} I_{1}(\gamma) \psi(d \gamma) & \leq \int_{S \times \Gamma}\left(\bar{\gamma} \log ^{+} \frac{\bar{\gamma}}{\kappa}\right) Q(s, d \gamma) \pi(d s) \\
& =\int_{S} E_{s}\left[\bar{\xi} \log ^{+} \frac{\bar{\xi}}{\kappa}\right] \pi(d s)=E_{\pi}\left[\bar{\xi} \log ^{+} \frac{\bar{\xi}}{\kappa}\right]
\end{aligned}
$$

which is finite by assumption.

The function $x \log ^{+} x$ is convex and therefore

$$
\sum_{i=1}^{n} \lambda_{i} x_{i} \log ^{+}\left(\sum_{i=1}^{n} \lambda_{i} x_{i}\right) \leq \sum_{i=1}^{n} \lambda_{i} x_{i} \log ^{+} x_{i}
$$

if $\sum_{i=1}^{n} \lambda_{i}=1, \lambda_{i} \geq 0$. For a fixed $\gamma$ we take $\lambda_{i}=\exp \left(-\alpha \tau_{i}(\gamma)\right) / \hat{\gamma}(\alpha)$ [and have $n=\gamma\left(S \times R_{+}\right)$] to obtain

$$
\begin{aligned}
I_{2}(\gamma) & \leq \hat{\gamma}(\alpha) \sum_{i=1}^{\infty} \frac{\exp \left(-\alpha \tau_{i}(\gamma)\right)}{\hat{\gamma}(\alpha)} E_{\gamma}\left[\bar{\xi}_{i} \log ^{+} \bar{\xi}_{i}\right] \\
& =\sum_{i=1}^{\infty} \exp \left(-\alpha \tau_{i}(\gamma)\right) E_{\gamma}\left[\bar{\xi}_{i} \log ^{+} \xi_{i}\right] .
\end{aligned}
$$


Hence

$$
\begin{aligned}
\int_{\Gamma} I_{2}(\gamma) \psi(d \gamma) & \leq \sum_{i=1}^{\infty} \int_{\Gamma} \exp \left(-\alpha \tau_{i}(\gamma)\right) E_{\gamma}\left[\bar{\xi}_{i} \log ^{+} \bar{\xi}_{i}\right] \psi(d \gamma) \\
& =\sum_{i=1}^{\infty} \int_{\Gamma} \exp \left(-\alpha \tau_{i}(\gamma)\right) E_{\sigma_{i}(\gamma)}\left[\bar{\xi} \log ^{+} \bar{\xi}\right] \psi(d \gamma) \\
& =\sum_{i=1}^{\infty} \int_{\Gamma \times S} \exp \left(-\alpha \tau_{i}(\gamma)\right) \delta_{\sigma_{i}(\gamma)}(d s) E_{s}\left[\bar{\xi} \log ^{+} \bar{\xi}\right] \psi(d \gamma) \\
& =\int_{\Gamma} E_{s}\left[\bar{\xi} \log ^{+} \bar{\xi}\right] \pi(d s)=E_{\pi}\left[\bar{\xi} \log ^{+} \bar{\xi}\right]<\infty
\end{aligned}
$$

and it is proved that $E_{\psi}\left[\bar{\eta} \log ^{+} \bar{\eta}\right]<\infty$. Now assume that $E_{\psi}\left[\bar{\eta} \log ^{+} \bar{\eta}\right]<\infty$. Because of the convexity we can apply Jensen's inequality which yields

$$
E_{\gamma}\left[\bar{\eta} \log ^{+} \bar{\eta}\right] \geq E_{\gamma}[\bar{\eta}] \log ^{+} E_{\gamma}[\bar{\eta}]=H(\gamma) \log ^{+} H(\gamma) .
$$

Hence

$$
\begin{aligned}
\infty & >E_{\psi}\left[\bar{\eta} \log ^{+} \bar{\eta}\right] \geq \int_{\Gamma} H(\gamma) \log ^{+} H(\gamma) \psi(d \gamma) \\
& =\int_{\Gamma \times \Gamma} \bar{\gamma} \log ^{+} \bar{\gamma} Q(s, d \gamma) \pi(d s) \\
& =\int_{\Gamma} E_{s}\left[\bar{\xi} \log ^{+} \bar{\xi}\right] \pi(d s)=E_{\pi}\left[\bar{\xi} \log ^{+} \bar{\xi}\right]
\end{aligned}
$$

and the proof is complete.

10. Convergence in $L^{1}$. The $L^{1}$-convergence of the macro process is explored by means of a certain martingale, introduced in Nerman (1984). Let $m x$ denote $x$ 's mother and define

$$
\mathscr{I}_{t}=\left\{x \in I: \tau_{m x}^{\prime} \leq t<\tau_{x}^{\prime}\right\},
$$

the set of macro individuals whose macro mothers are born before $t$ but who themselves are born after $t$. The intrinsic martingale is

$$
W_{t}=\sum_{x \in \mathscr{I}_{t}} \exp \left(-\alpha \tau_{x}^{\prime}\right) H\left(\xi_{x}\right) .
$$

From Jagers (1989) we know that the $x \log x$ condition implies that $W_{t}$ is uniformly integrable and hence there exists a random variable $W \geq 0$ on the macro space $\Gamma \times \bar{\Omega}^{I}$, such that $W_{t} \rightarrow W$ in $L^{1}\left(P_{\gamma}\right)$ as $t \rightarrow \infty$, for $\psi$-almost all $\gamma \in \Gamma$. The process will now be analyzed through the normed population size

$$
\zeta_{t}=\frac{e^{-\alpha t} Z_{t}^{\chi^{\prime}}}{H\left(\xi_{0}\right)} .
$$


With $\mathscr{I}_{t}$ as above, we get, for any $t_{0}<t$,

$$
\zeta_{t}=\frac{e^{-\alpha t}}{H\left(\xi_{0}\right)} \sum_{x<\mathscr{F}_{t_{0}}} \chi_{x}^{\prime}\left(t-\tau_{x}\right)+\sum_{x \in \mathscr{F}_{t_{0}}} \zeta_{t-\tau_{x}}(x) \exp \left(-\alpha \tau_{x}\right) \frac{H\left(\xi_{x}\right)}{H\left(\xi_{0}\right)}
$$

where $\zeta_{t-\tau_{x}}(x)=\zeta_{t-\tau_{x}} \circ S_{x}$ and $x<\mathscr{I}_{t_{0}}$ means that $x$ strictly precedes $\mathscr{I}_{t_{0}}$, that is, has descendants in $\mathscr{I}_{t_{0}}$ but does not itself belong to it. With $U_{x}\left(\gamma, \frac{t_{0}}{\omega}\right)$ $=\bar{\omega}_{x}$, the projection on the macro individual $x$ 's life, we define the $\sigma$-algebras

$$
\mathscr{F}_{t}=\mathscr{G} \times \sigma\left(U_{x}: x<\mathscr{I}_{t}\right),
$$

that is, the $\sigma$-algebras generated by the types and lives of the macro individuals strictly preceding $\mathscr{I}_{t}$.

The individual branching process is called uniformly integrable if $y_{t}$, the total number of individuals born up to time $t$, is uniformly integrable over its starting type, that is, if

$$
\sup _{s \in S} E_{s}\left[y_{t} ; y_{t}>c\right] \rightarrow 0, \quad c \rightarrow \infty
$$

for any fixed $t$.

We are ready for the main convergence result.

THEOREM 10.1. Consider a uniformly integrable, strictly Malthusian branching process with sibling dependencies such that $E_{\pi}\left[\bar{\xi} \log ^{+} \bar{\xi}\right]<\infty$. Let $\chi$ be bounded and the function $e^{-\alpha t} E_{s}[\chi(t)]$ directly Riemann integrable. Then there exists a random variable $w$ on $S \times \Omega^{I}$ such that

$$
e^{-\alpha t} z_{t}^{\chi} \rightarrow \frac{E_{\pi}[\hat{\chi}(\alpha)]}{\alpha \beta} w
$$

in $L^{1}\left(\tilde{Q}_{s}\right)$ for $\pi$-almost all $s \in S$.

PROOF. We first work with the macro process started from the children of the ancestor and show that $e^{-\alpha t} Z_{t}^{\chi^{\prime}}$ converges to $\left(E_{\pi}[\hat{\chi}(\alpha)] / \alpha \beta\right) W$.

Let $\chi^{\prime}(t)=\sum_{i=1}^{\infty} \chi_{i}\left(t-\tau_{i}\right)$ and assume that $\chi$ is bounded by $n$. Define $\chi^{n}(t)=\chi^{\prime}(t) 1_{[0, n]}(t)$ so that $\chi^{n}$ vanishes for $t \geq n$. It is $\chi^{n}$ that will be the characteristic under consideration. With $t_{0}=t-n$ in (10.1), we then have

$$
\zeta_{t}=\sum_{x \in \mathscr{F}_{t-n}} \zeta_{t-\tau_{x}^{\prime}}(x) \exp \left(-\alpha \tau_{x}^{\prime}\right) \frac{H\left(\xi_{x}\right)}{H\left(\xi_{0}\right)},
$$

since if $x<\mathscr{I}_{t-n}$, then $t-\tau_{x}^{\prime} \geq n$.

We will show that the $\zeta_{t}$ are uniformly integrable. From (8.2) we know that the $E_{\gamma}\left[\zeta_{t}\right]$ are uniformly bounded, and it remains to be shown that $\sup _{t} E_{\gamma}\left[\zeta_{t} ; A\right] \rightarrow 0$ as $P_{\gamma}(A) \rightarrow 0$. With $y_{t}(x)$ denoting the total number of 
individuals at time $t$ stemming from the individual $x$, we first note that

$$
Z_{t}^{\chi^{n}} \leq Z_{t}^{\chi^{\prime}}=\sum_{i=1}^{\xi_{0}(t)} z_{t-\tau_{i}\left(\xi_{0}\right)}^{\chi}(i) \leq n \sum_{i=1}^{\xi_{0}(t)} y_{t-\tau_{i}\left(\xi_{0}\right)}(i)
$$

and hence

$$
\zeta_{t-\tau_{x}^{\prime}}(x) \leq \frac{n}{H\left(\xi_{x}\right)} \exp \left(-\alpha\left(t-\tau_{x}^{\prime}\right)\right) \sum_{i=1}^{\xi_{x}\left(t-\tau_{x}^{\prime}\right)} y_{t-\tau_{x}^{\prime}-\tau_{i}\left(\xi_{x}\right)}(x i) .
$$

By (10.2) and the fact that

$$
H(\xi)=\sum_{i=1}^{\infty} \exp \left(-\alpha \tau_{i}(\xi)\right) h\left(\sigma_{i}(\xi)\right) \geq \kappa \hat{\xi}(\alpha),
$$

it now holds that

$$
\zeta_{t} \leq \frac{n}{\kappa} \sum_{x \in \mathscr{F}_{t-n}} \frac{\exp \left(-\alpha\left(t-\tau_{x}^{\prime}\right)\right)}{\hat{\xi}_{x}(\alpha)}\left(\sum_{i=1}^{\xi_{x}\left(t-\tau_{x}^{\prime}\right)} y_{n}(x i)\right) \exp \left(-\alpha \tau_{x}^{\prime}\right) \frac{H\left(\xi_{x}\right)}{H\left(\xi_{0}\right)},
$$

since if $x \in \mathscr{I}_{t-n}$, then $t-\tau_{x}^{\prime} \leq n$ and hence $y_{t-\tau_{x}^{\prime}-\tau_{i}\left(\xi_{x}\right)}(x) \leq y_{n}(x)$.

Now let $\varepsilon>0$ be given. By the uniform integrability of $y_{n}$, there exists a $\delta>0$ such that

$$
P\left(A \mid \mathscr{F}_{t-n}\right)<\delta \Rightarrow \sup _{x \in \mathscr{F}_{t-n}} E\left[y_{n}(x i) ; A \mid \mathscr{F}_{t-n}\right]<\varepsilon,
$$

where we also make use of the fact that if $x \in \mathscr{I}_{t-n}$, then the $\sigma_{x i}$ are measurable with respect to $\mathscr{F}_{t-n}$ (the individual types $\sigma_{x i}$ are determined by the type $\xi_{x}$ of the macro individual $x$, and this type is determined by the life of $x$ 's macro mother which belongs to $\mathscr{F}_{t-n}$ ). From this we see that

$$
\begin{gathered}
E\left[\frac{\exp \left(-\alpha\left(t-\tau_{x}^{\prime}\right)\right)}{\hat{\xi}_{x}(\alpha)} \sum_{i=1}^{\xi_{x}\left(t-\tau_{x}^{\prime}\right)} y_{n}(x i) ; A \mid \mathscr{F}_{t-n}\right] \\
=\frac{\exp \left(-\alpha\left(t-\tau_{x}^{\prime}\right)\right)}{\hat{\xi}_{x}(\alpha)} \sum_{i=1}^{\xi_{x}\left(t-\tau_{x}^{\prime}\right)} E\left[y_{n}(x i) ; A \mid \mathscr{F}_{t-n}\right] \\
<\frac{\exp \left(-\alpha\left(t-\tau_{x}^{\prime}\right)\right) \xi_{x}\left(t-\tau_{x}^{\prime}\right)}{\hat{\xi}_{x}(\alpha)} \varepsilon \leq \varepsilon
\end{gathered}
$$

if $P\left(A \mid \mathscr{F}_{t-n}\right)<\delta$, since, for any $\gamma \in \Gamma$,

$$
\frac{e^{-\alpha t} \gamma(t)}{\hat{\gamma}(\alpha)}=\frac{1}{\hat{\gamma}(\alpha)} \int_{0}^{t} e^{-\alpha(t-u)} e^{-\alpha u} \gamma(d u) \leq 1 .
$$

Now fix a $\delta_{0}$ and consider an $A \in \mathscr{S} \times A^{I}$ with $P_{\gamma}(A)<\delta_{0}$. With

$$
B=\left\{P\left(A \mid \mathscr{F}_{t-n}\right)<\delta\right\},
$$


we then get

and

$$
\delta P_{\gamma}\left(B^{c}\right) \leq P_{\gamma}\left(A \cap B^{c}\right) \leq P_{\gamma}(A)<\delta_{0}
$$

$$
\begin{aligned}
& \frac{\kappa H(\gamma)}{n} E_{\gamma}\left[\zeta_{t} ; A\right] \\
& \leq E_{\gamma}\left[E \left[\sum_{x \in \mathscr{F}_{t-n}} \exp \left(-\alpha \tau_{x}\right) H\left(\xi_{x}\right) \frac{\exp \left(-\alpha\left(t-\tau_{x}\right)\right)}{\hat{\xi}_{x}(\alpha)}\right.\right. \\
& \left.\left.\times \sum_{i=1}^{\xi_{x}\left(t-\tau_{x}\right)} y_{n}(x i) ; A \mid \mathscr{F}_{t-n}\right] ; B\right] \\
& +E_{\gamma}\left[E \left[\sum_{x \in \mathscr{F}_{t-n}} \exp \left(-\alpha \tau_{x}\right) H\left(\xi_{x}\right) \frac{\exp \left(-\alpha\left(t-\tau_{x}\right)\right)}{\hat{\xi}_{x}(\alpha)}\right.\right. \\
& \left.\left.\times \sum_{i=1}^{\xi_{x}\left(t-\tau_{x}\right)} y_{n}(x i) ; A \mid \mathscr{F}_{t-n}\right] ; B^{c}\right] \\
& \leq E_{\gamma}\left[\sum _ { x \in \mathscr { F } _ { t - n } } \operatorname { e x p } ( - \alpha \tau _ { x } ) H ( \xi _ { x } ) E \left[\frac{\exp \left(-\alpha\left(t-\tau_{x}\right)\right)}{\hat{\xi}_{x}(\alpha)}\right.\right. \\
& \left.\left.\times \sum_{i=1}^{\xi_{x}\left(t-\tau_{x}\right)} y_{n}(x i) ; A \mid \mathscr{F}_{t-n}\right] ; B\right] \\
& +E_{\gamma}\left[\sum _ { x \in \mathscr { F } _ { t - n } } \operatorname { e x p } ( - \alpha \tau _ { x } ) H ( \xi _ { x } ) E \left[\frac{\exp \left(-\alpha\left(t-\tau_{x}\right)\right)}{\hat{\xi}_{x}(\alpha)}\right.\right. \\
& \left.\left.\times \sum_{i=1}^{\xi_{x}\left(t-\tau_{x}\right)} y_{n}(x i) \mid \mathscr{F}_{t-n}\right] ; B^{c}\right] \\
& \leq \varepsilon E_{\gamma}\left[W_{t-n}\right]+\sup _{s \in S} E_{s}\left[y_{n}\right] E_{\gamma}\left[W_{t-n} ; B^{c}\right],
\end{aligned}
$$

where the first term can be made small since $E_{\gamma}\left[W_{t-n}\right]=E_{\gamma}[W]=H(\gamma)\left(W_{t}\right.$ is a martingale), and by choosing $\delta_{0}$ small enough $P_{\gamma}\left(B^{c}\right)$ will also be small and by the uniform integrability of $W_{t}$ (this is where the $x \log x$ condition appears) $E_{\gamma}\left[W_{t-n} ; B^{c}\right]$ will be uniformly small in $t$. Hence the $\zeta_{t}$ and thereby also the $e^{-\alpha t} Z_{t}^{\chi^{\prime}}$ are uniformly integrable. It can be proved that

$$
\zeta_{t}-E\left[\zeta_{t} \mid \mathscr{F}_{t-n}\right] \rightarrow 0
$$

in $P_{\gamma}$-probability for $\psi$-almost all $\gamma \in \Gamma$. This relies on the uniform integrability of the $y_{n}$ and is done exactly as in the proof of Theorem 7.2 in Jagers (1989) and is omitted here. With

$$
\rho_{n}=\frac{E_{\psi}\left[\hat{\chi}^{n}(\alpha)\right]}{\alpha B},
$$


we have that

$$
\begin{aligned}
& \frac{1}{H\left(\xi_{0}\right)}\left|E\left[\zeta_{t} \mid \mathscr{F}_{t-n}\right]-\rho_{n}\right| \\
& \quad \leq \int_{\Gamma \times R_{+}}\left|E_{\gamma^{\prime}}\left[\zeta_{t-u}\right]-\frac{\rho_{n}}{H\left(\xi_{0}\right)}\right| W_{t-n}\left(d \gamma^{\prime} \times d u\right)+\frac{\rho_{n}}{H\left(\xi_{0}\right)}\left|W_{t-n}-W\right|,
\end{aligned}
$$

where

$$
W_{t}(G)=\sum_{x \in \mathscr{I}_{t}} 1_{G}\left(\xi_{x}, \tau_{x}^{\prime}\right) \exp \left(-\alpha \tau_{x}^{\prime}\right) H\left(\xi_{x}\right)
$$

$G \in \mathscr{S} \times \mathscr{B}$. We already know that $W_{t} \rightarrow W$ in $L^{1}\left(P_{\gamma}\right)$ for $\psi$-almost all $\gamma \in \Gamma$, which takes care of the second term. To deal with the first term, introduce the special characteristic

$$
\chi^{G}(t)=e^{\alpha t} \int_{S} \int_{t}^{\infty} 1_{G}\left(\gamma^{\prime}, u\right) e^{-\alpha u} H\left(\gamma^{\prime}\right) \eta\left(d \gamma^{\prime} \times d u\right)
$$

for which it can be shown that

$$
W_{t}(G)=e^{-\alpha t} Z_{t}^{\chi^{G}}
$$

see Nerman (1984). It is an easy task to check that the Markov renewal conditions are satisfied so that Theorem 3 in Shurenkov (1992) can be applied and

$$
E_{\gamma}\left[W_{t}(G)\right] \rightarrow \frac{E_{\psi}\left[\hat{\chi}^{G}(\alpha)\right]}{\alpha B} H(\gamma) .
$$

Since $\left|E_{\gamma^{\prime}}\left[\zeta_{t-u}\right]-\rho_{n}\right|$ is bounded [a consequence of (8.1) together with the fact that the $E_{s}\left[e^{-\alpha t} z_{t}^{\chi}\right]$ are bounded; see the proof of Theorem 3 in Jagers (1992)],

$$
\begin{aligned}
\int_{\Gamma \times R_{+}}\left|E_{\gamma^{\prime}}\left[\zeta_{t-u}\right]-\rho_{n}\right| E_{\gamma}\left[W_{t-n}\left(d \gamma^{\prime} \times d u\right)\right] \\
\quad \leq \sup _{\left(\gamma^{\prime}, u\right) \in G}\left|E_{\gamma^{\prime}}\left[\zeta_{t-u}\right]-\rho_{n}\right| E_{\gamma}\left[W_{t}(G)\right]+M E_{\gamma}\left[W_{t}\left(G^{c}\right)\right],
\end{aligned}
$$

which can be made small for $t$ large by Egoroff's theorem. Thus

$$
\zeta_{t} \rightarrow \rho_{n} \frac{W}{H\left(\xi_{0}\right)}
$$

in $P_{\gamma}$-probability for $\psi$-almost all $\gamma \in \Gamma$. Hence we have that

$$
e^{-\alpha t} Z_{t}^{\chi^{n}} \rightarrow \rho_{n} W
$$

in $P_{\gamma}$-probability and, by the uniform integrability, also in $L^{1}\left(P_{\gamma}\right)$ for the characteristic $\chi^{n}$ under consideration [see, e.g., Ash (1972), page 297]. Finally, consider $\chi^{\prime}(t)=\sum_{i=1}^{\infty} \chi_{i}\left(t-\tau_{i}\right)$. With

$$
\rho=\frac{E_{\pi}[\hat{\chi}(\alpha)]}{\alpha \beta},
$$


it is easy to show that $\rho=E_{\psi}\left[\hat{\chi}^{\prime}(\alpha)\right] / \alpha B$ and we have that

$$
\begin{aligned}
E_{\gamma}\left[\left|e^{-\alpha t} Z_{t}^{\chi^{\prime}}-\rho W\right|\right] \leq & E_{\gamma}\left[\left|e^{-\alpha t} Z_{t}^{\chi^{\prime}}-e^{-\alpha t} Z_{t}^{\chi^{n}}\right|\right] \\
& +E_{\gamma}\left[\left|e^{-\alpha t} Z_{t}^{\chi^{n}}-\rho_{n} H(\gamma)\right|\right]+E_{\gamma}\left[\left|\rho_{n} H(\gamma)-\rho H(\gamma)\right|\right],
\end{aligned}
$$

where the first and third terms can be made small by choice of $n$ large and monotone convergence, and the second term is the case just treated.

Now define $w$ on $S \times \Omega^{I}$ through

$$
w\left(s, \omega_{I}\right)=W\left(\xi\left(\omega_{0}\right), \bar{\omega}\right) .
$$

By (3.1) and Lemma 3.1 it is clear that

$$
E_{s}\left[\left|e^{-\alpha t} z_{t}^{\chi}-w\right|\right] \leq E_{s}\left[e^{-\alpha t} \chi_{0}(t)\right]+\int_{\Gamma} E_{\gamma}\left[\left|e^{-\alpha t} Z_{t}^{\chi^{\prime}}-\rho W\right| Q(s, d \gamma)\right] .
$$

Since

$$
\psi(A)=\int_{S} Q(s, A) \pi(d s)
$$

it is clear that $Q(s, \cdot)$ is absolutely continuous with respect to $\psi(\cdot)$ for $\pi$-almost all $s \in S$. Therefore,

$$
\int_{\Gamma} E_{\gamma}\left[\left|e^{-\alpha t} Z_{t}^{\chi^{\prime}}-\rho w\right|\right] Q(s, d \gamma) \rightarrow 0
$$

for $\pi$-almost all $s \in S$.

By this theorem it is clear that the asymptotics of $e^{-\alpha t} z_{t}^{\chi}$ are established through conditions on the marginal reproduction measures $\mu(s, d r \times d t)$ alone. It is interesting to compare with an ordinary multitype process with the same marginals. Entities determined by the marginals alone remain the same; hence $\alpha, h$ and $\pi$ are the same in the sibling-dependent population as in the independent population. The constant $E_{\pi}[\hat{\chi}(\alpha)]$ is the same at least as long as individual characteristics are considered, that is, characteristics that only depend on an individual's own type and life, not on its progeny. For nonindividual characteristics, however, $E_{\pi}[\hat{\chi}(\alpha)]$ may differ substantially in the two populations compared, since the measure $\tilde{Q}_{\pi}$ clearly depends on the joint measures $P_{\gamma}$ (recall Example 2.1).

11. The stable population measure. The result in Theorem 10.1 indicates that the proportion of individuals counted by some characteristic $\chi$ stabilizes as time tends to $\infty$. This leads us to think of some kind of stable population, infinitely old and large with a stable composition of individuals. Such considerations lie behind the construction of the doubly infinite population space, introduced for single-type populations in Nerman and Jagers (1984). The multitype case is described in Jagers (1992) and, more extensively, in Jagers and Nerman (1992).

The doubly infinite population space is centered around an individual, Ego, thought of as sampled from all those born in an old exponentially 
growing population. The life and progeny of Ego can be viewed as an ordinary (or sibling-dependent, if that is the case) branching process with Ego as the ancestor. But now Ego also has a mother, a grandmother and so on, and she also has an age, thereby anchoring the population in real time. Therefore, let $Z_{-}=\{0,-1,-2, \ldots\}$, where Ego is 0 , her mother -1 and so on. From Ego's mother there will stem a population $-1 I$ and likewise for any individual $-j$. Therefore, let $J=Z_{-} \times I$ and define the doubly infinite population space as

$$
\boldsymbol{\Omega}=R_{+} \times N^{\infty} \times \Omega^{J},
$$

an element of which gives information about Ego's age, ancestry and the lives of all conceivable individuals in $J$. This space is endowed with the product $\sigma$-algebra denoted by $\mathscr{C}$. In order to describe the stable population measure on $(\boldsymbol{\Omega}, \mathscr{C})$, some random elements on this space are introduced.

Hence let $T_{0}$ denote Ego's age at sampling, $S_{0}$ her type and $R_{0}$ her rank. Also, $T_{1}$ is Ego's mother's age when she gave birth to Ego, $S_{1}$ her type, $R_{1}$ her rank and so on backwards. The life and progeny of Ego are denoted by $z_{0}$, $z_{1}$ denotes the life and progeny of Ego's mother except the life and progeny of Ego, $z_{2}$ the life and progeny of Ego's grandmother except Ego's mother's life and progeny and so on. Thus, after renumbering the population to tell which one of $-k$ 's children has been removed in order to play the role of $-(k-1)$, $z_{k}$ is the coordinate projection $\boldsymbol{\Omega} \rightarrow \Omega^{-k I}$.

DEFINITION 11.1. In an independent multitype branching process, the stable population measure is determined by

$$
\begin{aligned}
\mathbf{P}\left(z_{k} \in\right. & \left.A_{k}, T_{k} \in d t_{k}, S_{k} \in d s_{k}, R_{k}=i_{k}, k=0, \ldots, n\right) \\
= & E_{\pi}\left[\exp \left(-\alpha \tau_{i_{n}}\right) ; \sigma_{i_{n}} \in d s_{n}\right] \\
& \times E_{s_{n}}\left[\exp \left(-\alpha t_{n}\right) ; A_{n} \cap\left\{\sigma_{i_{n-1}} \in d s_{n-1}, \tau_{i_{n-1}} \in d t_{n}\right\}\right] \\
& \times \cdots \times E_{s_{1}}\left[\exp \left(-\alpha t_{1}\right) ; A_{1} \cap\left\{\sigma_{i_{0}} \in d s_{0}, \tau_{i_{0}} \in d t_{1}\right\}\right] \\
& \times P_{s_{0}}\left(A_{0}\right) \alpha \exp \left(-\alpha t_{0}\right) d t_{0},
\end{aligned}
$$

where $A_{k} \in \mathscr{A}^{I}$.

This definition has a number of consequences described in Jagers and Nerman (1992). For instance, the stable population measure gives Ego an age which is exponentially $(\alpha)$ distributed and independent of everything else and a type distributed according to $\pi$.

The main difference in a multitype sibling-dependent population is that even if an individual's marginal reproduction only depends on her type, there are still dependencies between siblings; the conditional independence structure in independent multitype populations now only exists on the macro level. We want to study the individual stable population but for this it is necessary to invoke the macro process. Therefore, any defining relation of the stable population measure should take into account the following two considerations: (1) the whole reproduction processes should be used as information carriers between generations, and (2) the lives and progenies of whole sibling 
groups should be considered rather than individuals. Hence let $S_{k}, T_{k}$, and $R_{k}$ be as above and define $\Gamma_{0}$ as the reproduction process according to which Ego was born and $\Gamma_{1}$ the process according to which Ego's mother was born and so on. Further let $Z_{0}$ be the lives and progeny of Ego and her siblings, $Z_{1}$ the lives and progeny of Ego's mother and her siblings except the lives and progeny of Ego and her siblings and so on.

DEFINITION 11.2. In a multitype branching process with sibling dependencies, the stable population measure is determined by

$$
\begin{aligned}
\mathbf{P}\left(Z_{k} \in\right. & \left.B_{k}, T_{k} \in d t_{k}, \Gamma_{k} \in d \gamma_{k}, R_{k}=i_{k}, k=0, \ldots, n\right) \\
= & \exp \left(-\alpha \tau_{i_{n}}\left(\gamma_{n}\right)\right) \psi\left(d \gamma_{n}\right) \\
& \times E_{\gamma_{n}}\left[\exp \left(-\alpha t_{n}\right) ; B_{n} \cap\left\{\xi_{i_{n}} \in d \gamma_{n-1}, \tau_{i_{n-1}}\left(\xi_{i_{n}}\right) \in d t_{n}\right\}\right] \\
& \times \cdots \times E_{\gamma_{1}}\left[\exp \left(-\alpha t_{1}\right) ; B_{1} \cap\left\{\xi_{i_{1}} \in d \gamma_{0}, \tau_{i_{0}}\left(\xi_{i_{1}}\right) \in d t_{1}\right\}\right] \\
& \times P_{\gamma_{0}}\left(B_{0}\right) \alpha \exp \left(-\alpha t_{0}\right) d t_{0},
\end{aligned}
$$

where $B_{k} \in \mathscr{A}^{I_{1}}$.

Note. Of course, this is how $\mathbf{P}$ looks also in an independent population but then, because of the independencies, the $P_{\gamma}$ are equal to the products of their marginals.

It can be shown that the stable population measure indeed appears as a limit of averages in a growing population. This is done almost exactly as in, for example, Jagers and Nerman (1992), only with some care that has to be taken because of the dependencies. The exact results and proofs may be found in Olofsson (1994). Here we only give a quick description of $\mathbf{P}$ through some of its consequences.

12. Properties of the stable population measure. It is natural to compare the stable population measure in a sibling-dependent population with its independent analog, that is, the stable population measure that arises from an ordinary independent multitype process with the same individual marginals as the sibling-dependent one.

It is obvious from Definition 11.2 that $T_{0}$ is still exponential $(\alpha)$ and independent of everything else. Some trivial calculations also yield that Ego's individual properties are the same in the two populations. Actually, even more than this holds, for instance, the following two propositions which are exactly the same as in the independent case.

Proposition 12.1. The sequence $S_{0}, S_{1}, \ldots$ of types backwards from Ego is a Markov chain with transition probabilities

$$
\mathbf{P}\left(S_{n+1} \in d s \mid S_{n}=r\right)=\pi(d s) \frac{\hat{\mu}(s, d r)}{\pi(d r)} .
$$


Proposition 12.2. The sequence of types and interbirth times backwards from Ego, $\left(S_{n}, T_{n}\right)_{n=0}^{\infty}$, is a Markov renewal process with transition kernel

$$
\mathbf{P}\left(S_{n+1} \in d s, T_{n+1} \in d t \mid S_{n}=r\right)=\pi(d s) \frac{e^{-\alpha t} \mu(s, d r \times d t)}{\pi(d r)} .
$$

Hence a lot of properties of a population remain unaffected by sibling dependencies. That a lot of properties are also affected is best realized by considering Example 2.1 where, for instance, we have $\mathbf{P}(B)=1 / 3$ and $\mathbf{P}\left(A^{c} \cap B\right)=2 / 81$ in the independent population, but $\mathbf{P}(B)=29 / 81$ and $\mathbf{P}\left(A^{c} \cap B\right)=0$ in the sibling-dependent population.

13. General local dependencies. A natural extension is to allow dependencies also between first cousins, second cousins and so on [see, e.g., Kubitschek (1967)]. Let us start by introducing some terminology and convenient notation. Call a branching process $n$-dependent if individuals who are $k$ th cousins reproduce independently if $k \geq n$ but possibly dependently if $k<n$. By convention, siblings are considered as 0 th cousins. Then $0-$ dependence means independence, 1-dependences means sibling dependence, 2 -dependence means that individuals who are either siblings or first cousins reproduce dependently and so forth. There are no dependencies between generations other than through the types. The term "local" in the heading obviously means local in the family tree. We will give a quick description of how to treat local dependencies with the techniques from previous sections without delving into the details.

There will be a hierarchy of macro individuals and we define a $k$-macro individual to consist of a group of individuals each two of whom are $j$ th cousins for some $j=0,1, \ldots, k$, where $0 \leq k \leq n$.

There is now a sequence of life spaces $\Omega_{0}, \Omega_{1}, \ldots$, where

$$
\Omega_{k+1}=\Omega_{k}^{\infty},
$$

$\Omega_{0}$ being the individual life space. Likewise, there will be a sequence of type spaces, $\Gamma_{0}, \Gamma_{1}, \ldots$, where

$$
\Gamma_{k+1}=\mathscr{N}\left(\Gamma_{k} \times R_{+}\right),
$$

and, in the previous notation, $\Gamma_{0}=S$ and $\Gamma_{1}=\Gamma$. Let $\sigma_{i}: \Gamma_{k+1} \rightarrow \Gamma_{k}, k=$ $1,2, \ldots$, so that $\sigma_{i}\left(\gamma_{k+1}\right)$ is the type of the $i$ th $k$-macro individual in a $(k+1)$-macro individual. The sequence of probability measures $P_{0}, P_{1}, \ldots$ on $\Omega_{0}, \Omega_{1}, \ldots$ is then such that the $i$ th $k$-macro individual in a $(k+1)$-macro individual reproduces according to the marginal

$$
P_{k}\left(\sigma_{i}\left(\gamma_{k+1}\right), A\right)=\int_{\Omega_{k+1}} 1_{A}\left(p_{i}\left(\omega_{k+1}\right)\right) P_{k+1}\left(d \omega_{k+1}\right),
$$

the projections $p_{i}$ being defined in the obvious way. Again, it will be convenient to consider the measures induced by the reproduction processes. There- 
fore, define

$$
Q_{k}\left(\gamma_{k}, d \gamma_{k+1}\right)=P_{k}\left(\gamma_{k},\left\{\eta^{k} \in d \gamma_{k+1}\right\} \mid\left\{\eta^{k} \in \Gamma_{k+1}\right\}\right),
$$

with the obvious notation $\eta^{0}, \eta^{1}, \ldots$, where $\eta^{0}=\xi$. These measures give rise to the sequence of reproduction kernels $M_{0}, M_{1}, \ldots$, where $M_{0}=\mu$ and $M_{1}=M$, and the next lemma is obvious.

Lemma 13.1. In an n-dependent branching process, the kernels $M_{0}$, $M_{1}, \ldots, M_{n}$ all define the same Malthusian parameter. Further, the kernels $\hat{M}_{0}, \hat{M}_{1}, \ldots, \hat{M}_{n}$ are either all conservative or all not.

Now let $\tau_{i}: \Gamma_{k} \rightarrow R_{+}$so that $\tau_{i}\left(\gamma_{k}\right)$ is the time point for the $i$ th point in $\gamma_{k}$. Under conservativity assumptions, there will also be a sequence of functions $H_{0}, H_{1}, \ldots, H_{n}$ and a sequence of measures $\psi_{0}, \psi_{1}, \ldots, \psi_{n}$ such that $H_{k}$ and $\psi_{k}$ are invariant for $M_{k}$ and such that, for instance,

$$
H_{k+1}\left(\gamma_{k+1}\right)=\bar{\gamma}_{k+1}=\sum_{i=1}^{\infty} \exp \left(-\alpha \tau_{i}\left(\gamma_{k+1}\right)\right) H_{k}\left(\sigma_{i}\left(\gamma_{k+1}\right)\right) \text {. }
$$

In the previous notation, $H_{0}=h, H_{1}=H, \psi_{0}=\pi$ and $\psi_{1}=\psi$. The mean convergence is obvious; the problems of $L^{1}$-convergence are twofold: only the individual process is assumed uniformly integrable, and only the function $h=H_{0}$ is assumed to be bounded away from 0 . Therefore, we cannot just copy the previous proofs by noting that a group of $n$-macro individuals can be viewed as a sibling group consisting of $(n-1)$-macro individuals. However, the proofs can be modified also to this setting.

We start by proving that all the $x \log x$ conditions are equivalent. For that purpose note that

$$
\bar{\eta}^{k+1}=\sum_{i=1}^{\infty} \exp \left(-\alpha \tau_{i} \bar{\eta}_{i}^{k}\right)
$$

Here $\eta_{i}^{k}$ is the point process stemming from the $i$ th $k$-macro individual, and $\bar{\eta}^{k}$ can be defined from $\eta^{k}$ as $\bar{\eta}$ was defined from $\eta$. The $x \log x$ conditions are

$$
E_{\psi_{k}}\left[\bar{\eta}^{k} \log ^{+} \bar{\eta}^{k}\right]<\infty
$$

for $k=0,1,2, \ldots, n$, and we state the following result.

LEMMA 13.2. Either

$$
E_{\psi_{k}}\left[\bar{\eta}^{k} \log ^{+} \bar{\eta}^{k}\right]<\infty
$$

for $k=0,1,2, \ldots, n$, or

$$
E_{\psi_{k}}\left[\bar{\eta}^{k} \log ^{+} \bar{\eta}^{k}\right]=\infty
$$

for $k=0,1,2, \ldots, n$. 
PROOF. Let

$$
\begin{array}{r}
f\left(\gamma_{k}\right)=\sum_{i_{k-1}, \ldots, i_{0}} \exp \left(-\alpha \tau_{i_{k-1}}\left(\gamma_{k}\right)\right) \exp \left(-\alpha \tau_{i_{k-2}}\left(\sigma_{i_{k-1}}\left(\gamma_{k}\right)\right)\right) \\
\times \cdots \times \exp \left(-\alpha \tau_{i_{0}}\left(\sigma_{i_{1}}\left(\cdots \sigma_{i_{k-1}}\left(\gamma_{k}\right)\right) \cdots\right)\right)
\end{array}
$$

and note that

$$
\begin{gathered}
\bar{\gamma}_{k}=\sum_{i_{k-1}=1}^{\infty} \exp \left(-\alpha \tau_{i_{k}-1}\left(\gamma_{k}\right)\right) H_{k-1}\left(\sigma_{i_{k-1}}\left(\gamma_{k}\right)\right) \\
=\sum_{i_{k-1}, i_{k-2}} \exp \left(-\alpha \tau_{i_{k-1}}\left(\gamma_{k}\right)\right) \exp \left(-\alpha \tau_{i_{k-2}}\left(\sigma_{i_{k-1}}\left(\gamma_{k}\right)\right)\right) \\
=\cdots=\sum_{i_{k-1}, \ldots, i_{0}} \exp \left(-\alpha \tau_{i_{k-1}}\left(\gamma_{k}\right)\right) \exp \left(-\alpha \tau_{i_{k-2}}\left(\sigma_{i_{k-1}}(\gamma k)\right)\right) \\
\quad \times \cdots \times \exp \left(-\alpha \tau_{i_{0}}\left(\sigma_{i_{1}}\left(\cdots \sigma_{i_{k-1}}\left(\gamma_{k}\right)\right) \cdots\right)\right) \\
\left.\quad \times h\left(\sigma_{i_{0}}\left(\cdots \sigma_{i_{k-1}}\left(\gamma_{k}\right)\right) \cdots\right)\right) \\
\geq \kappa f\left(\gamma_{k}\right) . \quad
\end{gathered}
$$

Having established this inequality, the rest of the proof is carried out by carefully copying the proof of Lemma 9.1 .

Finally, we state the analog of Theorem 10.1. The proof is essentially the same with the function $f$ from (13.2) playing the role of $\hat{\xi}(\alpha)$. It is omitted but may be found in Olofsson (1994).

THEOREM 13.3. Consider an n-dependent nonlattice, uniformly integrable and strictly Malthusian branching process such that $E_{\pi}\left[\bar{\xi} \log ^{+} \xi\right]<\infty$. Let $\chi$ be bounded and the function $e^{-\alpha t} E_{s}[\chi(t)]$ directly Riemann integrable. Then there exists a random variable $w$ such that

$$
e^{-\alpha t} z_{t}^{\chi} \rightarrow \frac{E_{\pi}[\hat{\chi}(\alpha)]}{\alpha \beta} w
$$

as $t \rightarrow \infty$ in $L^{1}\left(\tilde{Q}_{s}\right)$ for $\pi$-almost all starting types $s \in S$.

Acknowledgment. This paper is based on parts of my Ph.D. thesis, and I would like to thank my former supervisor Professor Peter Jagers for valuable support and discussions.

\section{REFERENCES}

Ash, R. B. (1972). Real Analysis and Probability. Academic Press, London.

BROBERG, P. (1987). Sibling dependencies in branching populations. Ph.D. dissertation, Göteborg Univ.

CRump, K. S. and Mode, C. J. (1969). An age-dependent branching process with correlations among sister cells. J. Appl. Probab. 6 205-210. 
JAGERS, P. (1989). General branching processes as Markov fields. Stochastic Process. Appl. 32 $183-212$.

JAGERS, P. (1992). Stabilities and instabilities in population dynamics. J. Appl. Probab. 29 $770-780$.

JAGERS, P. (1995). Branching processes as population dynamics. Bernoulli 1 191-200.

JAGERS, P. and Nerman, O. (1992). The asymptotic composition of supercritical multi-type branching populations. Technical report, Dept. Mathematics, Chalmers Univ. Technology and Göteborg Univ.

KubitscheK, H. E. (1967). Cell generation times: ancestral and internal controls. Proc. Fifth Berkeley Symp. Math. Statist. Probab. 4 549-572. Univ. California Press, Berkeley.

Nerman, O. (1984). The growth and composition of supercritical branching populations on general type spaces. Technical report, Dept. Mathematics, Chalmers Univ. Technology and Göteborg Univ.

Nerman, O. and JAGERS, P. (1984). The stable doubly infinite pedigree process of supercritical branching populations. Z. Wahrsch. Verw. Gebiete 65 445-460.

Olofsson, P. (1994). General branching processes with local dependencies. Ph.D. dissertation, Göteborg Univ.

Powell, E. (1955). Some features of the generation times of individual bacteria. Biometrika 42 $16-44$.

Shurenkov, V. M. (1989). Ergodicheskie Protsessy Markova. Nauka, Moscow.

SHuRENKov, V. M. (1992). Markov renewal theory and its application to Markov ergodic processes. Technical report, Dept. Mathematics, Chalmers Univ. Technology and Göteborg Univ.

\author{
Department of Mathematics \\ Chalmers University of Technology \\ S-412 96 GÖTEBORG \\ SWEDEN \\ E-mail:petero@math.chalmers.se
}

